\title{
Nested Sliding Window Protocols with Packet Fragmentation
}

\author{
Gerald W. Shapiro \\ and \\ Harry G. Perros
}

Center for Communications and Signal Processing Electrical and Computer Engineering Department North Carolina State University

CCSP TR-89/15

October 1989 


\title{
Nested Sliding Window Protocols With Packet Fragmentation'
}

\author{
Gerald W. Shapiro \\ Department of Management Science \\ VPI \& SU \\ Blacksburg, VA 24061-0235 \\ Harry G. Perros \\ Department of Computer Science \\ North Carolina State University \\ Raleigh, NC 27695-8206
}

\begin{abstract}
A single-hop OSI structured network with multiple layers of sliding window flow control and packet fragmentation between layers is analyzed. An approximation algorithm is presented to hierarchically reduce the network to a single queue whose performance characteristics represent the original network. Network transmission characteristics are restricted to Erlang distributions. Validation against exact and simulation results showed that the approximation algorithm has a satisfactory error level.
\end{abstract}

\section{INTRODUCTION}

Most modern communication network protocols are designed using a layered structure along the lines of the OSI model [1]. A common means of implementing the required sequencing, pacing, congestion control, and data integrity functions of a protocol layer is a sliding window protocol [1]. Under such a protocol each data packet at a given layer in the sender is given a number in the range 0 to $W-1 . W$ is the window size of this layer. Upon successful receipt of a data packet, the receiving protocol peer generates an acknowledgment packet for the sender, indicating the number of the received data packet. Once the acknowledgment is received by the sender, the packet number can be re-used to send another packet. If at any time the sender has $W$ unacknowledged packets outstanding, that sender is blocked and cannot pass another packet to the layer below it until an acknowledgment for one of the outstanding

1 Supported in part by AIRMICS via the North Carolina State University Center for Communications and Signal Processing, and by the Rome Air Force Development Center Grant E-21-669-58 through the Georgia Institute of Technology. 
packets arrives. There are a number of variants on this basic scheme used in practice, (see [2]).

Within a given hop, more than one protocol layer may have a sliding window scheme. In such a situation each protocol layer will have its own window size and packet numbers, and the sliding window schemes are such that one is imbedded within the other. We refer to this structure as nested window protocols.

Each layer may have a maximum size data unit it can process, the maximum packet size of that layer. When a layer at the sending station, say layer $i$, has a larger maximum packet size than the layer below it, (layer $i-1)$, it may be necessary to split the packet from layer $i$ into a number of smaller packets. This process is referred to as fragmentation of the higher layer data packet. The peer layer $\mathrm{i}-1$ at the receiving station must reassemble the fragments before passing the original data up to the layer $i$ above it.

Of interest to network designers are the throughput and delay performance characteristics of networks using sliding window protocols. Since the sending layers will periodically be blocked, the effective transmission capability of the network is less than the raw transmission rate.

Even simplified models of the sliding window protocol have resisted efforts to date to find exact closed form solutions for the performance measures, (although numerical techniques involving the analysis of the underlying Markov chain may be applied to fairly small examples.) Current analysis depends upon approximation techniques.

Sliding window protocols were analyzed originally (see [3]-[6]) using a closed queueing network with population $W$. This implies that packets do not enqueue at a blocked sender, but rather that packets arriving to a blocked sender are "lost". Such models do not accurately represent the total delay characteristics of packet delivery. Lam [7] presents a general modelling framework which can model a sliding window protocol under this loss assumption. Thomasian and Bay [8] review a number of related techniques. 
The models described above deal with only a single layer of sliding window control, and do not include packet fragmentation. Goto, Takahashi, and Hasegawa [9] and Akyildiz [10] analyze a model which limits the number of packets allowed at each intermediate station between the sender and receiver by blocking the downstream neighbor of a full station, in addition to limiting the number of packets in the end to end connection by the loss assumption. This comes closer to modelling nested sliding window schemes, but no acknowledgment mechanism is modeled, nor is packet fragmentation.

An analysis useful for predicting the maximum throughput of a sliding window controlled link is presented by Kleinrock and Kermani [11]. In this analysis there is always a packet waiting for a returning acknowledgment. Fragmentation and nesting are not considered.

Reiser [12] and Thomasian and Bay [13], use a flow-equivalent server technique to model the sliding window link as a single server queue with state dependent service rate. In this approach the acknowledgment packets do not explicitly appear in the approximating queue; the effect of delays due to all sequence numbers in use is accounted for in the delivery service time of the equivalent server. While these models do not consider fragmentation, they do explicitly model the delay of packets enqueued at a blocked sender.

Modelling approaches similar to the above are used by Gihr and Kuehn [14], Varghese, Chou and Nilsson [15], and Schwartz [16]. In Gihr and Kuehn's analysis the characteristics of the physical transmission process are obtained from analysis of a LAN, in a hierarchical model. They also consider group arrivals to the sliding window controlled layer, modelling fragmentation from a higher layer. Both [15] and [16] note that the method is not very accurate in the face of group arrivals.

Three recent papers also address the need for a multi-layer analysis of communications networks. Mitchell and Lide [17] present a general framework which uses a closed queveing network, (ala [6]), to model sliding window control. Murata and Takagi [18] consider a token ring LAN operating underneath a sliding window protocol layer. Again a closed queueing 
network model is used for the sliding window layer. Neither paper addresses packet fragmentation.

Fdida, Perros, and Wilk [19] present a methodology for analyzing arbitrary configurations of sliding window controlled networks. Both nested and series configurations are allowed; however, packets may not be fragmented, and are presumed to arrive singly. Each layer of sliding window control is reduced to a state-dependent infinite server queue without explicit acknowledgments, using a flow-equivalence methodology. Queueing delays for transmission when all sequence numbers are in use is accounted for in the mean service time of the infinite server. The method is hierarchical, reducing the lowest layers first.

In this paper a hierarchical method is presented for analyzing nested sliding window controlled layers. Each layer with sliding window control is reduced to a single queve without explicit acknowledgments. The simple nature of the equivalent queue facilitates analysis of multiple layers. Unlike other papers, specific attention is given in this analysis to fragmentation and reassembly. The analysis here is restricted to a single-hop (no intermediate stations) connection.

In Section II, the model of nested layers of sliding window protocols is presented. Section III gives the approximation methodology, and in Section IV the approximations are compared to exact and simulation results. Finally, the conclusions are given in Section V.

\section{A Model for Nested Layers of Sliding Window Flow Control}

Let us consider for a moment, a single-hop communication link with one sliding window flow control as shown in Figure 1. 


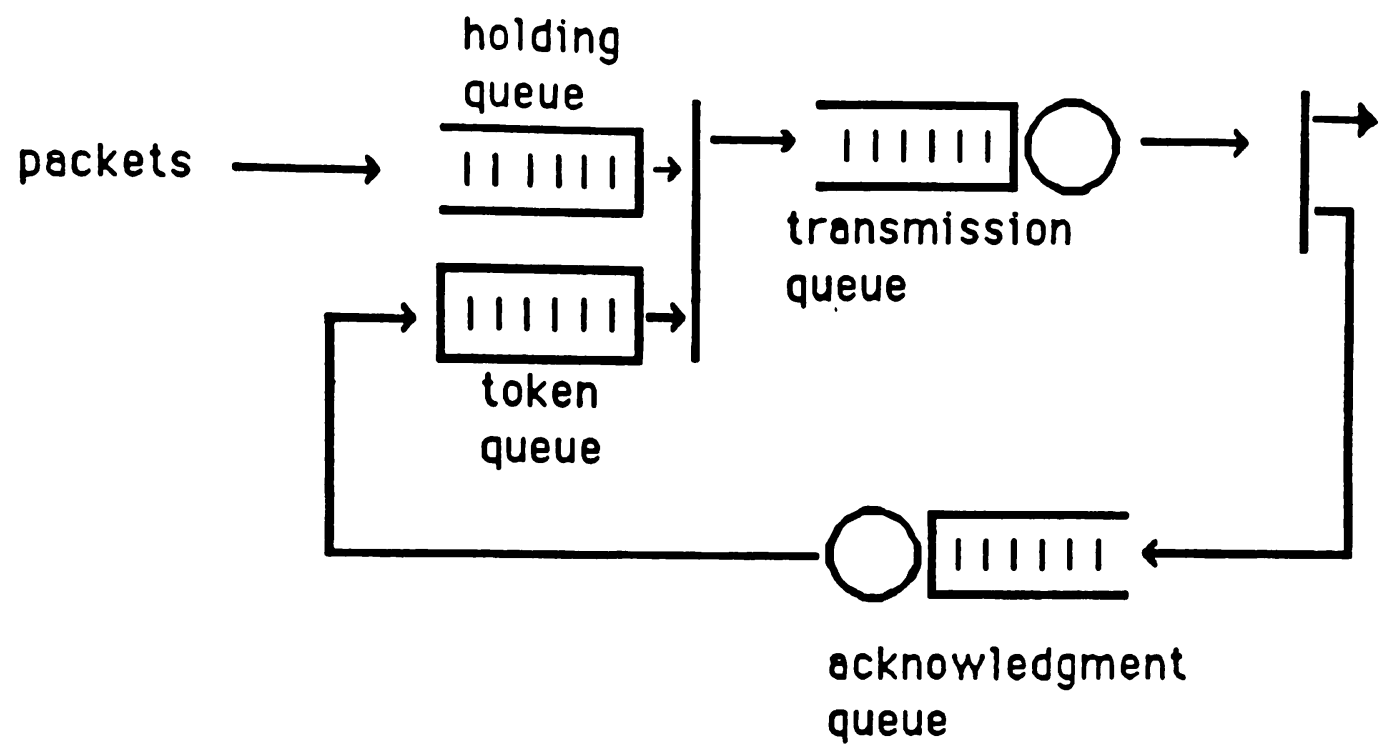

Figure 1 - Single Sliding Window Flow Control

Packets arrive at the holding queue. If there is a token available at the token queue, a packet takes the token and proceeds to the transmission queue. Upon service completion the packet leaves the system and the token joins the acknowledgment queue. After completion of its service, the token joins the token queve. Packets that arrive at the system when the token queue is empty are forced to wait in the holding queve. The number of tokens is set equal to the maximum window size.

In order to represent the window flow control in a simple manrier, we make use of the following two symbols

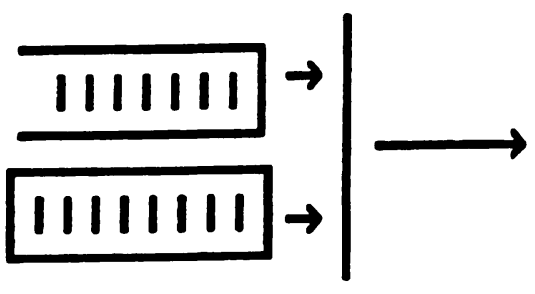

join

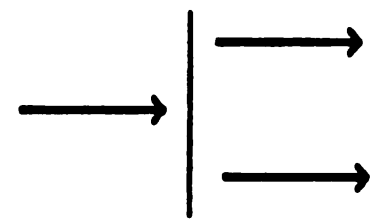

split 
(We note that these symbols are similar to those used in Fdida, Perros, and Wilk [19]). The join symbol depicts the following operation: when both queves contain a customer each. the two customers instantaneously depart from their respective queves and merge into a single customer. The split symbol depicts the operation where a customer arriving at this point is split into two entities.

Now, let us consider the above single-hop communication link with two layers of nested sliding window flow control and packet fragmentation/reassembly, as shown in Figure 2.
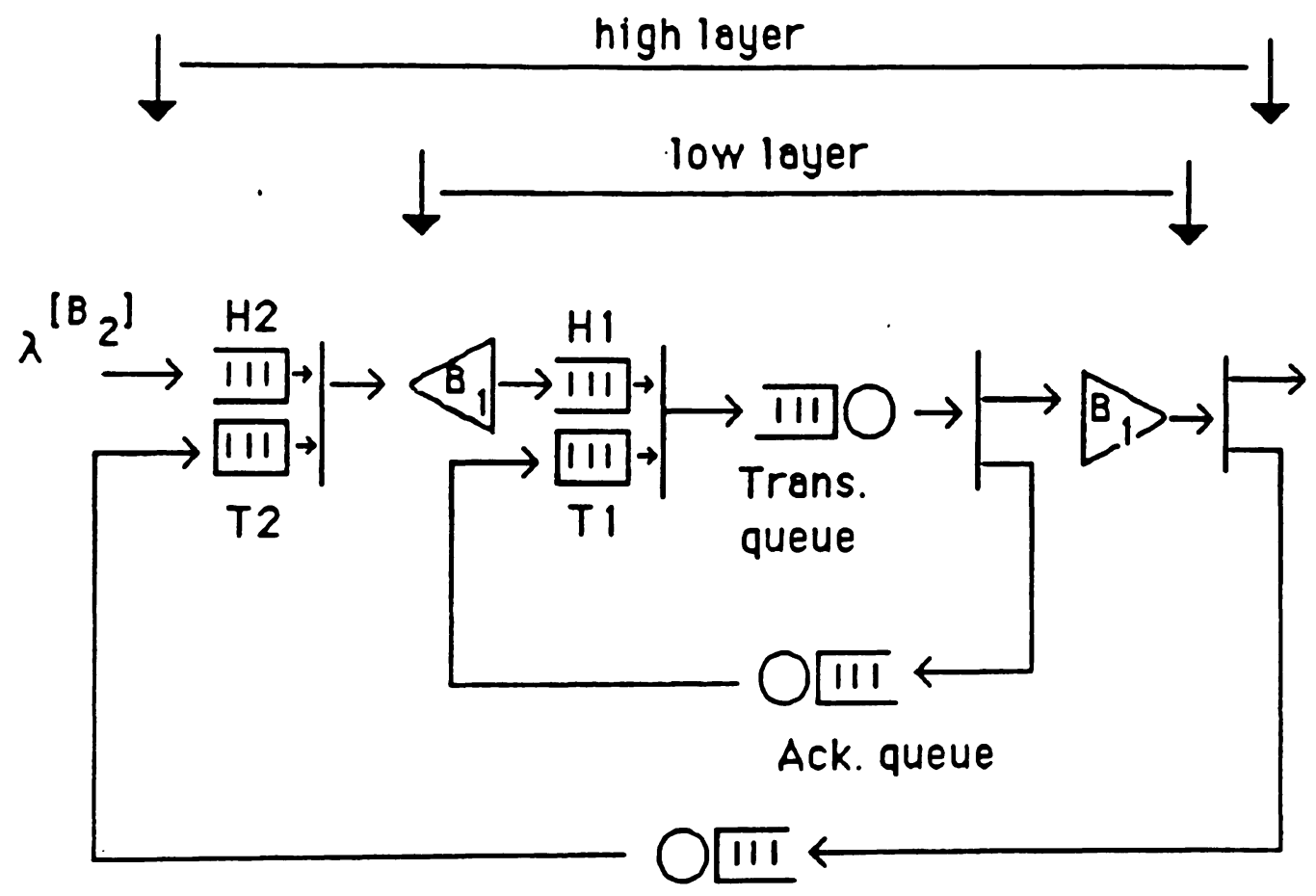

Ack. queue

Figure 2 - The Model for the Nested Two-Layered Sliding Window Flow Control

In order to represent packet fragmentation and reassembly we use the following two symbols. 


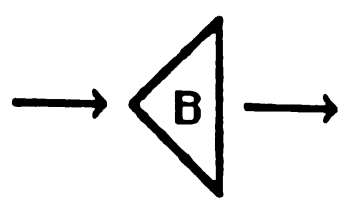

fragmentation

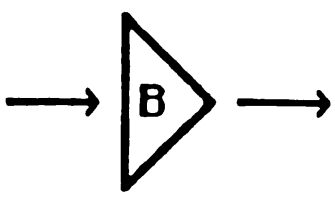

reassembly

The fragmentation symbol indicates the operation where a packet upon arrival at that point is split into $B$ subpackets. This operation is assumed to take zero time. The reassembly symbol indicates a buffer where subpackets wait. When the Bth subpacket arrives, all the $B$ subpackets are instantaneously assembled into a single packet which is released immediately

For presentation purposes, we shall refer to the outside window flow control in Figure 2 as the high layer, and to the inside window flow control as the low layer. Packets arrive at the high layer according to a Poisson process with rate $\lambda$. An arriving packet is immediately fragmented into $B_{2}$ high layer packets. Each of these packets requires a token from the token queve 72 in order to enter the low layer. Those high layer packets for which there is no token available are forced to wait in the holding queve $\mathrm{H} 2$. Upon entry to the low layer, a high layer packet is immediately fragmented into $B$, low layer packets. Each of these packets is then subjecled to window flow control using token queue T1. They are then transmitted to the receiver one at a time. An acknowledgment for a low layer packet is generated immediately upon receipt of the packet. High layer packets are acknowledged upon receipt of $B$, low layer packets comprising the original high layer packet Let $W_{2}$ and $W_{1}$ be the window size for the high and low layer respectively.

We assume that packets arrive in the order in which they were sent, as would be the case in a virtual circuit protocol. The transmission time is modelled as a single server queue with exponentially distributed server time. Retransmission of packets is ignored, assuming that the mean transmission time measures average time to successfully deliver a packet. We also assume an infinite buffer at layer 2, and sufficient buffer space at layer 1 to hold $W_{2} B_{1}$ packets. (the most that could be at this layer). We note that in real-life there are other service delays 
in addition to the transmission time These service delays have been ignored as we wish to focus on the interaction between the sliding window flow control and packet fragmentation/reassembly. These delays can be easily incorporated approximately seeing that the model is analyzed using a hierarchical decomposition method.

Although the above described model has only two layers of sliding window control, it is of sufficient generality to demonstrate the method of analysis proposed here. The goal is to reduce an arbitrary number of nested layers of sliding window control to a single server queue which represents the performance characteristics of the nested layers. This is done in a hierarchical manner. We first analyze the low layer, as shown in Figure 1, in order to construct a flow-equivalent queve. (This equivalent queve is obtained assuming a Poisson arrival process. Upon arrival, a packet is immediately fragmented into $B_{1}$ subpackets.) Then, in Figure 2. we replace the low layer by its equivalent queue, thus obtaining a queueing model similar to the one shown in Figure 3, where the transmission queue represents the equivalent queue.

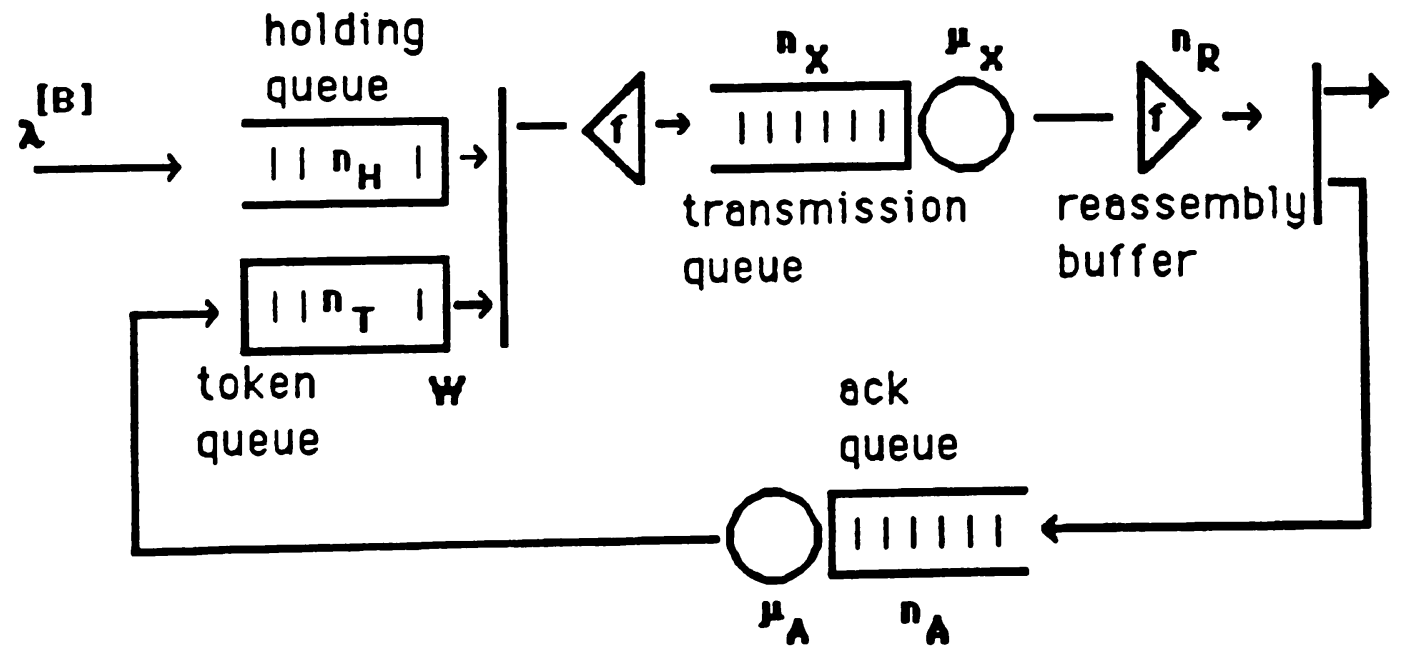

Figure 3 - The Model of a Sliding Window Flow Control With Packet Fragmentation and Reassembly

Next. we reduce the network in Figure 3 to a single queue, which will represent the characteristics of the original network. If there is another protocol layer above the two depicted in Figure 2, then this new equivalent queve is inserted as the transmission path in that layer. 
Note that unlike the methodology in [12] and related papers, this study does not use a statedependent service rate in the equivalent queue. Given exponential transmission times, each equivalent queue will be of the Erlang type.

It is evident that in order to be able to tackle any number of nested layers of sliding window flow control we need to be able to construct a flow equivalent queue of the queuing system shown in Figure 3. This is taken up in the following section.

\section{Reduction to a Single Queue}

Let us consider the model shown in Figure 3. Arrivals are in batches of $B$. The inter-arrival time between two batches is exponentially distributed with mean $1 / \lambda$. The window size is $W$. When a packet goes through window flow control it is fragmented into $f$ packets. We shall refer to the packets in the holding queue as high layer packets, and the fragmented packets inside the window flow control as low layer packets. The service times at the transmission queue and the acknowledgment queue are exponentially distributed with parameter $\mu_{x}$ and $\mu_{A}$ respectively.

Let us define the following notation:

$n_{H}$ : number of high layer packets in holding queve

$$
n_{T} \text { : number of tokens in token queue }
$$

$n_{A}$ : number of tokens in acknowledgment queue

$n_{X}$ : number of lower layer packets in transmission queue

$n_{R}$ : number of lower layer packets in reassembly buffer

$n_{s}$ : number of high layer packets undelivered.

(i.e., the number of packets"in the system").

$$
n_{S}=n_{H}+\frac{1}{f}\left(n_{X}+n_{R}\right)
$$

Given the triple $\left(n_{S}, n_{A}, n_{R}\right)$ other quantities above can be calculated using the following relations:

$$
\begin{gathered}
n_{X}=f \min \left(n_{S}, W-n_{A}\right)-n_{R}, \\
n_{T}=W-n_{A}-\left(n_{X}+n_{R}\right) / f, \\
n_{H}=n_{S}-\left(n_{X}+n_{R}\right) / f .
\end{gathered}
$$


The triple $\left(n_{S}, n_{A}, n_{R}\right)$ then suffices as the state of the system. We will denote a specific state by $(i j, k)$.

Let $p_{I J, k}$ be the probability of finding the system in state $(i, j, k)$ in steady state. The steady state probability balance equations which determine $p_{i, k}$ are:

$$
\begin{aligned}
& \lambda p_{0,0,0}=\mu_{A} p_{0,1,0} \\
& n_{S}=0 \quad\left(\lambda+\mu_{A}\right) p_{0, j, 0}=\mu_{A} p_{0, j+1,0}+\mu_{X} p_{1, j-1, f-1}, j=1,2, \ldots, W-1 \\
& \left(\lambda+\mu_{A}\right) p_{0, W, 0}=\mu_{X} p_{1, W-1, f-1} \\
& \left(\lambda+\mu_{X}\right) p_{i, 0,0}=\mu_{A} p_{i, 1,0} \\
& \left(\lambda+\mu_{X}\right) p_{i, 0, k}=\mu_{A} p_{i, 1, k}+\mu_{X} p_{i, 0, k-1}, \quad k=1,2, \ldots, f-1 \\
& \left(\lambda+\mu_{A}+\mu_{X}\right) p_{i, j, 0}=\mu_{A} p_{i, j+1,0}+\mu_{X} p_{i+1, j-1, f-1}, \quad j=1,2, \ldots, W-1 \\
& 0<n_{S}<B \quad\left(\lambda+\mu_{A}+\mu_{\chi}\right) p_{i, j, k}=\mu_{A} p_{i, j+1, k}+\mu_{X} p_{i, j, k-1} \text {. } \\
& j=1,2, \ldots, W-2 k=1,2, \ldots, f-1 \\
& \left(\lambda+\mu_{A}+\mu_{X}\right) p_{i, W-1, k}=\mu_{X} p_{i, W-1, k-1}, \quad k=1,2, \ldots, f-1 \\
& \left(\lambda+\mu_{A}\right) p_{1, W, 0}=\mu_{X} p_{i+1, W-1, f-1} \\
& \left(\lambda+\mu_{X}\right) p_{i, 0,0}=\mu_{A} p_{i, 1,0}+\lambda p_{i-B, 0,0} \\
& \left(\lambda+\mu_{X}\right) p_{i, 0, k}=\mu_{A} p_{i, 1, k}+\mu_{X} p_{i, 0, k-1}+\lambda p_{i-B, 0, k}, \quad k=1,2, \ldots, f-1 \\
& \left(\lambda+\mu_{A}+\mu_{X}\right) p_{1, j, 0}=\mu_{A} p_{i, j+1,0}+\mu_{X} p_{i+1, j-1, f-1}+\lambda p_{i-B, j, 0}, \quad j=1,2, \ldots, W-1 \\
& n_{S} \geq B \quad\left(\lambda+\mu_{A}+\mu_{X}\right) p_{i, j, k}=\mu_{A} p_{i, j+1, k}+\mu_{X} p_{i j, k-1}+\lambda p_{i-B, j, k} . \\
& j=1,2, \ldots, W-2 \quad k=1.2, \ldots, f-1 \\
& \left(\lambda+\mu_{A}+\mu_{X}\right) p_{1, W-1, k}=\mu_{X} p_{i, W-1, k-1}+\lambda p_{1-B, W-1, k}, \quad k=1,2, \ldots, f-1 \\
& \left(\lambda+\mu_{A}\right) p_{I, W, 0}=\mu_{x} p_{i+1, W-1, f-1}+\lambda p_{i-B, W, 0}
\end{aligned}
$$

It is desired to reduce the sliding window controlled network of Figure 3 to a single server queve, and to that end we look at the probabilities $P_{11}$

$$
P_{i}=\sum_{j=0}^{W} \sum_{k=0}^{t-1} p_{i, j, k}
$$


$P_{1}$ is the probability of having $i$ high layer packets in the entire system. We will work from the rate equations governing $P_{i}$, in order to construct this equivalent queue.

The rate equations governing $P$, are obtained by summing all of the rate equations (1) with $i$ as a subscript on the left hand side. These equations are, (with like terms on left and right hand sides cancelled),

$$
\begin{gathered}
\lambda P_{0}=\mu_{x} \sum_{j=0}^{W-1} p_{1, j, f-1} \\
\lambda P_{i}=\mu_{x} \sum_{j=0}^{W-1} p_{1+1, f, f-1}-\mu_{x} \sum_{j=0}^{W-1} p_{i j, f-1}, \quad 0<i<B . \\
\lambda P_{i}=\lambda P_{i-B}+\mu_{x} \sum_{j=0}^{W-1} p_{i+1, j, f-1}-\mu_{x} \sum_{j=0}^{W-1} p_{i, f-1}, \quad i \geq B
\end{gathered}
$$

We see that the summation $\sum_{j=0}^{W-1} p_{i, j, f-1}$ plays an important role. This quantity is the probability of being in those states from which an immediate transition to a state with one less high layer packet in the system is possible when there are $i$ packets in the system. (The number of high layer packets in the system only decreases when the final fragment of an original high layer packet completes transmission. This only occurs when the number in the reassembly buffer is $f-1)$.

It is desirable to have a set of rate equations for $P_{i}$ which do not depend upon the "internal" parameters of the lower layer, $n_{A}$ and $n_{R}$. Dependence of the rate equations $(3)$ on the reassembly buffer population can be eliminated by making the assumption (for $i>0$ ),

$$
\sum_{j=0}^{W-1} p_{i j, k}=\sum_{j=0}^{W-1} p_{i j, k}
$$

for each pair $k, k^{\prime} \in\{0,1, \ldots, f-1\}$. Assumption (4) states that it is equally likely to find any of the possible reassembly buffer populations when there are $i>0$ packets in the system, as long 
as $n_{A}<W$, i.e., as long as there are packets in the transmission queue. Since the mean time spent with $k$ in the reassembly buffer, (given that a packet is in transmission), is $1 / \mu_{x}$ for each possible value of $k$, assumption (4) seems reasonable.

(In fact, while it is true that

$$
\sum_{i=1}^{\infty} \sum_{j=0}^{W-1} p_{i, j, k}=\sum_{i=1}^{\infty} \sum_{j=0}^{W-1} p_{i j, k}
$$

(4) is not true; however, (4) proves to be an adequate assumption).

Using assumption (4) and (2) we have

$$
P_{i}=p_{1, W, 0}+\sum_{k=0}^{f-1} \sum_{j=0}^{W-1} p_{i, j, k}=p_{i, W, 0}+f \sum_{j=0}^{W-1} p_{i, f-1}
$$

thus

$$
\sum_{j=0}^{W-1} p_{i j, f-1}=\left(P_{i}-p_{i, W, 0}\right) / f
$$

(5) shows another quantity that proves pivotal in the analysis of the sliding window protocol; $\boldsymbol{p}_{i, w, 0}$, is the probability that all tokens are in the acknowledgement queue, and $i$ high layer packets are in the system. Define $a_{i}$ as the conditional probability of having all the tokens in the acknowledgment queue given $i$ high layer packets in the system, i.e.,

$$
a_{i}=\frac{p_{i, w, 0}}{P_{i}}
$$

Combining (5) and (6), we have

$$
\sum_{j=0}^{W-1} p_{i, j, f-1}=P_{i}\left(1-a_{i}\right) / f
$$


Substituting this expression for $\sum_{j-0}^{W-1} p_{i j, f-1}$ into (3), we note that under assumption (4), (3) is the set of rate equations for a bulk arrival queue with state dependent exponential service rate $\frac{\mu_{x}}{f}\left(1-a_{1}\right)$.

The values $a_{i}$ can be calculated from the solution to the rate equations (3). There is no known closed form solution to these equations, thus the solution must rely upon a numerical technique. Other authors who have used a flow equivalent server methodology to model sliding window protocols use an approximation procedure to generate the $a_{i}$, for $i=1,2, \ldots, W$, and assume that $a_{w+n}=a_{w}$ for any $n>0$, (see [12]-[18]). The $a_{\text {, }}$ are not computed explicitly in these papers, but rather the system throughput is analyzed for the closed system consisting of the transmission and acknowledgement queves when there are $i$ packets in the closed network. Since this throughput is equal to $\mu_{x}\left(1-a_{1}\right)$, the computation of $a_{1}$ is implicit. We have observed empirically that the results of this approximation are not good for a bulk arrival system, while they are satisfactory for Poisson arrivals, (see Fdida, Perros, Wilk [19]).

This study takes another approach to simplifying this system. We shall replace the state dependent service rates $\frac{\mu_{x}}{f}\left(1-a_{i}\right)$ with a single, state independent service rate, which is, in some sense, an average of the state dependent rates. This results in a fairly simple approximation of the original system.

Let us look at the nature of the values $a_{i}$. Figure 4 shows a plot of $a_{i}$ vs. $i$, for a typical system. 


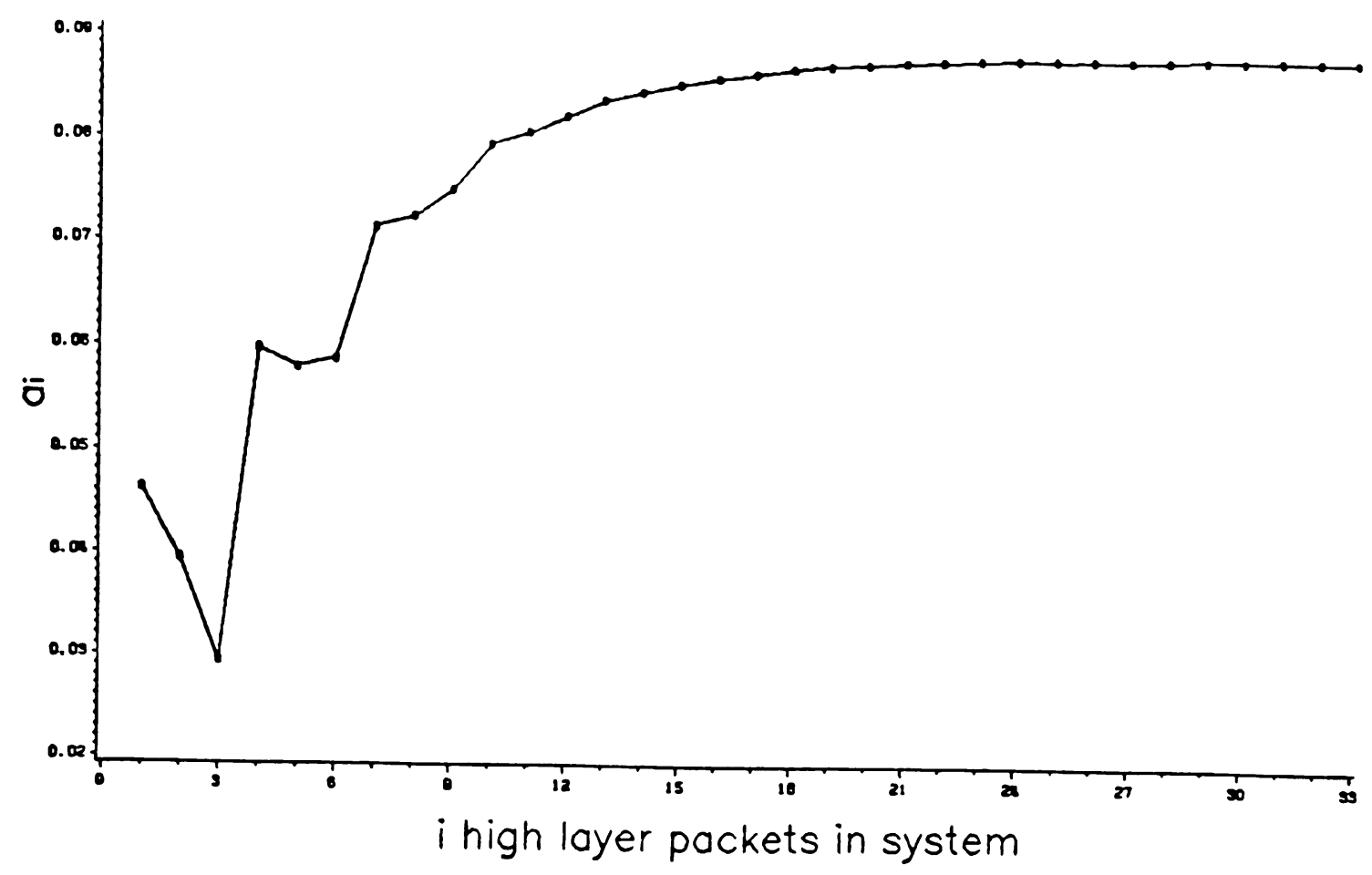

Figure 4 - a,vs.i

We observe that the values tend towards a constant as $i$ gets large. This behavior was exhibited for every choice of parameter values of the system.

Let us refer to the settling down of $a$, to a constant as $i$ increases as a state-asymptotic property. (This is to distinguish from time asymplotic behavior). That all systems examined show this behavior is not surprising. The state transition structure of the model is aperiodic. When there are a large number of packets in the system, the system will behave much like a closed model with $W$ tokens circulating. in that each token returning to the sending station will find a packet waiting. As the holding queue is being depleted, the tokens will be continuously moving through the system and we would expect that the distribution of the tokens between the acknowledgment and transmission queues will reach a statistical equilibrium. Let us denote the state-asymptotic value of $a_{1}$ by $a_{\infty}$.

Also note that $a$, is smallest for $i=B$. This behavior is also typical of all data sets. The situation where $i=B$ can occur when a batch arrival of size $B$ occurs to an empty system. At 
this point, since the holding queue has been empty, the sending station will have had a chance to "stockpile" some tokens, and thus the probability of having all tokens in the acknowledgment queve will be relatively low. (The state $i=B$ can also be reached by a transmission completion with B+1 packets in the system, but for systems with utilization not close to one. this event is less likely than arrival to an empty system.)

To construct a single queue approximation to the model under study with state independent service rate, we would like to find a value $\bar{a}$ which is an average of the $a_{i}$. This average value should be less than $a_{\infty}$, and greater that $a_{0}$.

Let us look at estimating $a_{\infty}$. the upper bound on $\overline{\mathbf{a}}$. An obvious approach is to use a closed network version of the original network with $W$ packets circulating. This network is shown in Figure 5 .

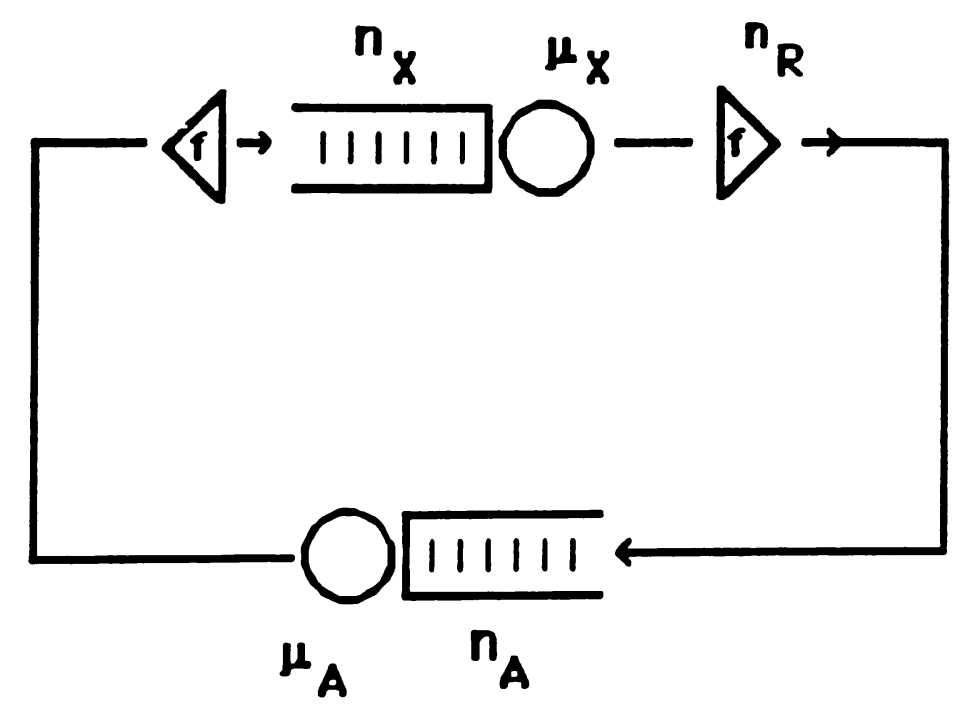

Figure 5 - Closed Model Used in Approximation Procedure

The rate diagram for this closed network is shown in Figure 6 . for the case where $f=3$ and $W=4$. 


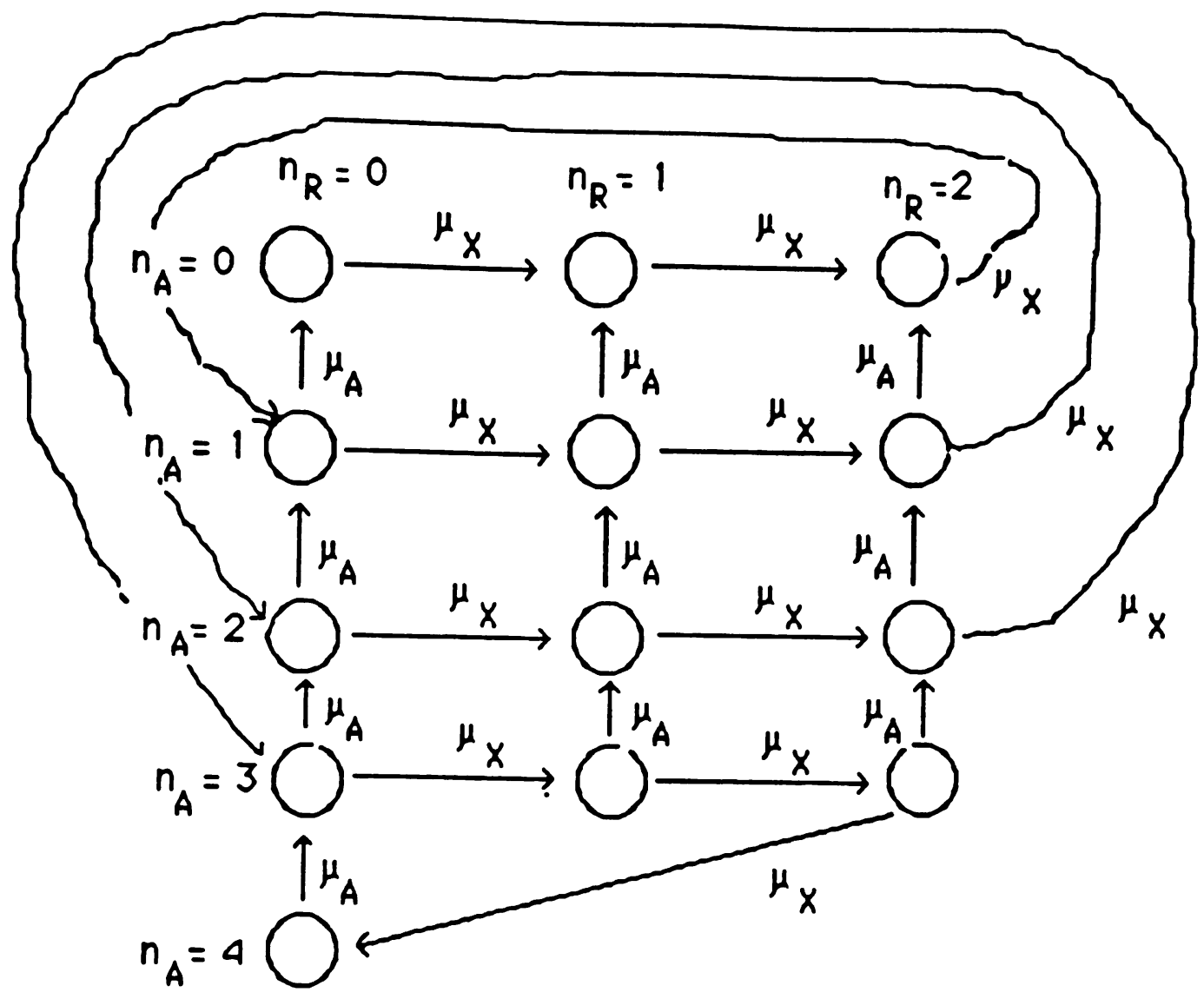

Figure 6 - Rate Diagram of Model Shown in Figure 5 for $W=4, f=3$.

Take for the state of the closed system the pair $\left(n_{A} . n_{R}\right)$. Denote the probability of state $(j . k)$ by $y_{\text {,.k }}$

Since there are a finite number of states in this model, the state probabilities of this system are rather easily solved for. It seems that the probability $y_{w, 0}$ should be a good estimate of $a_{m}$. as the original system under saturation behaves much like a closed network; however, it turns out that $y_{w, 0}$ over-estimates $a_{\infty}$ for every choice of parameters examined.

An explanation for this discrepancy comes from comparing the nature of the closed system to the original. Note that in the original system each transrisission completion when $n_{R}=f-1$ takes us from a state where $n_{s}=n+1$ to a state where $n_{s}=n$. In the closed sys- 
tem there is no explicit value of $n_{s}$. It is assumed that $n_{s}$ is "large", and that the number in the acknowledgment queue is increased at the rate at which transmission completions occur when $n_{R}=f-1$, and decreased at the rate at which acknowledgment completions occur. Thus the closed model estimates for large $n_{s}$ that the probability flux into state $(n, j+1,0)$ due to a transmission completion is $\mu_{x} p_{n, j, 1-1}$, where $n$ is a "large" value of $n_{s}$. In the original system. the flux into state $(n j+1,0)$ due to transmission completions is $\mu_{x} p_{n+1,1,1-1 .}$ If $p_{n j, 1-1}$ and $p_{n+1,1,-1}$ were equal, then the closed model would accurately predict the probability distribution of the acknowledgment queue for large $n_{s}$; however, in general these probabilities are not equal.

If we look at the state asymptotic behavior of the probabilities $P_{i}$, we note that the value of $P_{i+1} / P_{i}$ tends to a constant ratio as $i$ gets large. (see Figure 7 ).

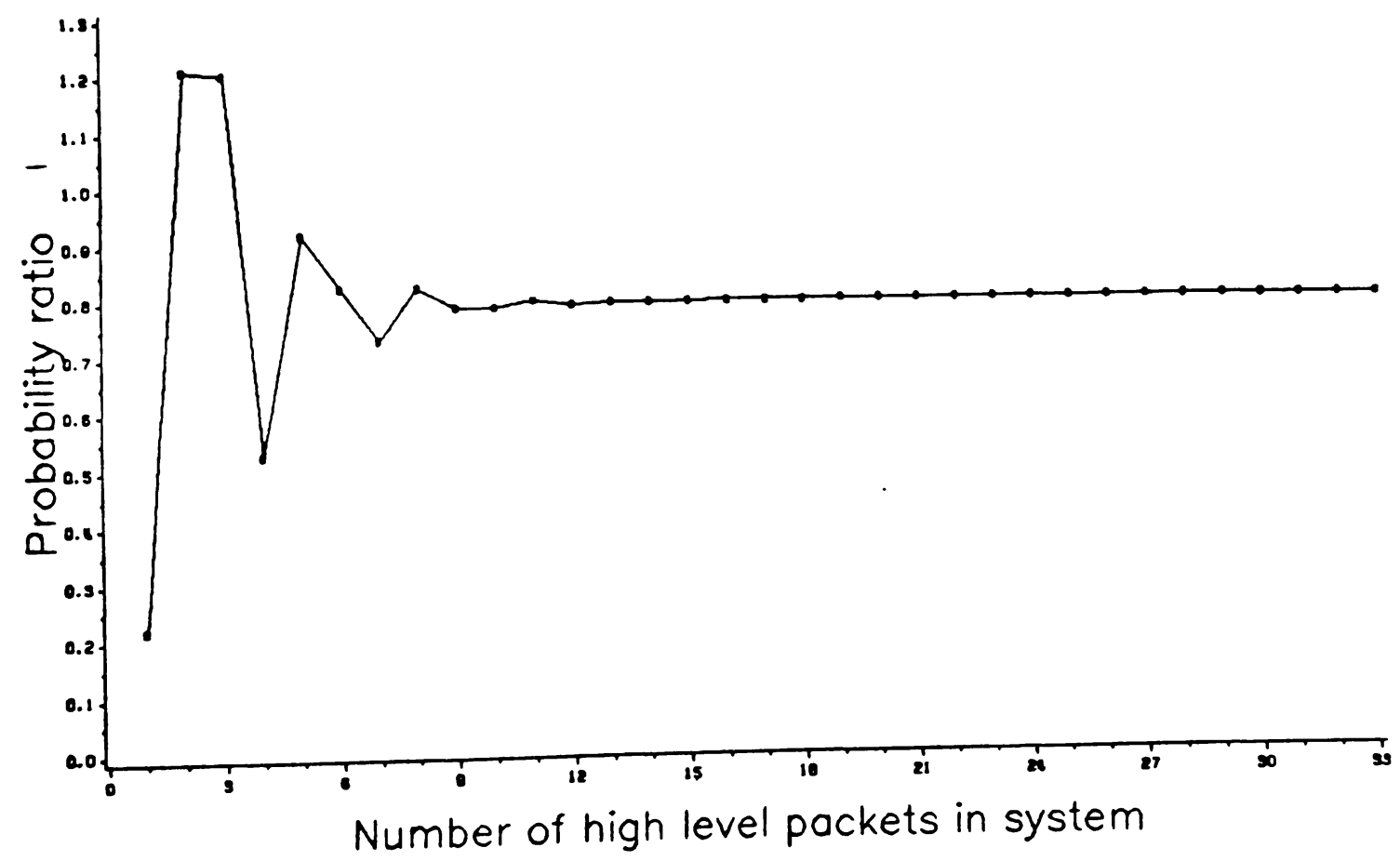

Figure $7-P_{i, 1} / P_{i}$ vs. $i$

This phenomenon is observed for all of the test cases run. We thus post that for $i$ large

$$
P_{1+1}=\gamma_{\infty} P_{i}
$$


for some $\gamma_{\infty} \in(0,1)$. A formula for $\gamma_{\infty}$ comes from summing the aggregate rate equations (3) for $i=0,1,2, \ldots, N+B$, which gives us (after simplification)

$$
\lambda \sum_{i=N+1}^{N+B} P_{i}=\mu_{X} \sum_{j=0}^{W-1} p_{N+B+1, f,-1}
$$

From (7) and (assuming $N$ is large) assumption (8), (9) becomes, (after simplification),

$$
a_{N+B+1}=1-\frac{\lambda}{\left(\mu_{X} / f\right)} \frac{1-\gamma_{\infty}^{B}}{\gamma_{\infty}^{B}\left(1-\gamma_{\infty}\right)} .
$$

We see that assumption (8) implies the existence of $a_{\infty}$, since the right-hand side above is independent of $N$. We thus have an equation relating $a_{\infty}$ and $\gamma_{\infty}$

$$
a_{\infty}=1-\frac{\lambda}{\left(\mu_{x} / f\right)} \frac{1-\gamma_{\infty}^{B}}{\gamma_{\infty}^{B}\left(1-\gamma_{\infty}\right)} .
$$

Another equation relating $a_{\infty}$ and $\gamma_{\infty}$ is obtained from an analysis of the closed network, (Figure 5). We have already seen that empirical evidence indicates that there is a constant ratio between state probabilities for large $n_{s}$. Let us modify the rate at which tokens enter the acknowledgment queue in the closed model in order to reflect more accurately the true dynamics of the system. Since the only service completions which result in a token entering the acknowledgment queue are those which occur when $n_{R}=f-1$, we will replace the rate $\mu_{x}$ at which transmission completions occur in these states by $\gamma_{\infty} \mu_{x}$, and leave the other service rates alone.

The probability balance equations for this modified system are:

$$
\begin{aligned}
& \gamma_{\infty} \mu_{X} y_{W-1, f-1}=\mu_{A} y_{W, 0} \\
& n_{A}=W-1 \quad \mu_{X} y_{W-1, f-2}=\left(\mu_{A}+\gamma_{\infty} \mu_{X}\right) y_{W-1, f-1} \\
& \mu_{X} y_{W-1, k}=\left(\mu_{A}+\mu_{X}\right) y_{W-1, k+1}, \quad k=f-3, \ldots, 0
\end{aligned}
$$




$$
\begin{aligned}
& n_{A}=W-2, \ldots, 1 \quad \gamma_{\infty} \mu_{x} y_{j, f-1}=\mu_{A} \sum_{k=0}^{t-1} y_{j+1, k} \\
& \mu_{X} y_{j, f-2}=\left(\mu_{A}+\gamma_{\infty} \mu_{X}\right) y_{j, f-1}-\mu_{A} y_{j+1, f-1} \\
& \mu_{X} y_{j, k}=\left(\mu_{A}+\mu_{X}\right) y_{j, k+1}-\mu_{A} y_{j+1, k+1}, k=f-3, \ldots, 0 \\
& n_{A}=0 \quad \gamma_{\infty} \mu_{x} y_{0, f-1}=\mu_{A} \sum_{k=0}^{t-1} y_{1, k} \\
& \mu_{X} y_{0, f-2}=\gamma_{\infty} \mu_{X} y_{0, f-1}-\mu_{A} y_{1, f-1} \\
& \mu_{X} y_{0, k}=\mu_{X} y_{0, k+1}-\mu_{A} y_{1, k+1}, k=f-3, \ldots, 0
\end{aligned}
$$

Equations (11) can be solved by noting that the closed network on which the rate equations are based can be cast as a Coxian server with a state dependent arrival rate. Marie, [20], has developed an algorithm for solving for the state probabilities of such a queue.

Let us see how the closed network can be described as a Coxian server with state dependent arrival rates. Let $n_{c}=W-n_{A} . n_{c}$ is the number of high layer packets in the transmission queue, (including the high layer packet in service). Arrivals to the transmission queue occur at rate $\mu_{A}$ whenever the acknowledgment queue is not empty, thus the state dependent arrival process to the transmission queue, denoted by $\lambda\left(n_{c}\right)$ is given by

$$
\lambda\left(n_{C}\right)=\left\{\begin{array}{cl}
\mu_{A}, & n_{C}=0,1, \ldots, W-1 \\
0, & n_{C} \geq W
\end{array}\right.
$$

The transmission server can be thought of as a Coxian-f server of high layer packets. A high layer packet beginning service at the transmission server requires $f$ sequential exponential service phases before completing service. In the model with modified rates, (i.e. (11)), the first f- 1 service phases have rate $\mu_{x}$ and the last phase has rate $\gamma_{\infty} \mu_{x}, y_{w, 0}$, the probability of having all tokens in the acknowledgment queue in the closed model, is equal to the probability that $n_{c}=0$ in the Coxian server with state dependent arrival rate (12). This probability can be calculated from the algorithm in [20]. 
Using the solution from (11) for $y_{w, 0}$ as an estimate of $a_{\infty}$ together with (10) gives us two relationships between $a_{\infty}$ and $\gamma_{\infty}$. Solving for the pair $a_{\infty}, \gamma_{\infty}$ which simultaneously satisfies both relations resulted in a consistent under-estimate of $a_{\infty}$; however, this under-estimate proved empirically to be a fairly good choice for $\bar{a}$. Furthermore, it was empirically found that it suffices to carry out a couple of iterations of the algorithm used to find the simultaneous solution to (10) and (11). Thus, the following algorithm proves to give a good value for $\bar{a}$.

\section{Approximation Algorithm}

1. Let $\gamma_{\infty}=1$. Solve (11) for $a_{\infty}$.

2. Use the value of $a_{\infty}$ from 1) to calculate a new $\gamma_{\infty}$, using (10).

3. Using $\gamma_{\infty}$ from 2), calculate a new $a_{\infty}$ from the closed network equations (11). Use this value of $a_{\infty}$ for $\bar{a}$.

The approximation algorithm gives a good estimate for the average service rate of high layer packets, $\frac{\mu_{x}}{f}(1-a)$. It remains to determine the type of server to be used for the approximate queue. The model of Figure 3 has a transmission mechanism which must complete $f$ exponentially distributed transmissions to deliver a high layer packet. The transmission time for the high layer packet thus has an Erlang-f distribution. In the approximation procedure, we used an Erlang-h server with mean service rate $\frac{\mu_{x}}{f}(1-a)$ to represent the transmission network as seen by the higher layer.

The number of stages, $h$, in the approximate Erlang server is determined as follows. If $f$ is 2 or $3, h=2$ is used. This choice is based on comparison of experiments with $h=1,2$, and 3 in the approximation algorithm against exact solutions computed by Neuts' matrix-geometric procedure, [21]. For any $f>3, h=3$ is used. Since the sliding window mechanism introduces variability into the effective delivery time distribution beyond that of the transmission time distribution, less than $f$ stages are used in the approximate model, since the Erlang-k family of distributions has increasing variance as $k$ decreases. The exception to this rule is when $f=2$. In this case the Erlang-2 distribution and the exponential, or Erlang-1, gave comparable results. The number of stages in the approximate model is limited to three, since the Erlang-k distributions become less distinct as $k$ increases. It is hoped that using no more than three stages will suffice. 
Limiting the number of stages in the approximate Erlang server also helps to keep the computational burden of the approximation scheme down when more than two nested layers of sliding window protocol are analyzed. Consider the network of Figure 8(a). This network has three layers of sliding window protocol. Analyzing the performance of this network hierarchically, we would first reduce the lowest layer to a single exponential server. We use the approximation algorithm to compute the service rate of this server, analyzing the lowest layer model with arrivals at rate $\lambda B_{1} B_{2}$ in batches of size $B_{3}$. After this step we have the approximate network of Figure 8(b). Now we use the approximation algorithm to reduce the lowest layer of sliding window protocol in this network, using an arrival process of rate $\lambda B_{1}$ with batches of size $B_{2}$. This results in the network of Figure $8(c)$, where the Erlang-h server has $h=2$ or 3, depending upon the magnitude of $B_{3}$.

The network of Figure $8(\mathrm{c})$ appears to be a model which we have not yet considered. It differs from the model of Figure 3 by having an Erlang rather than an exponential server. From a modelling point of view, however, these two networks have the same structure. In the model of Figure $3 f$ exponential services must be completed to deliver a high layer packet. From the point of view of the higher layer, the fragmentation and re-assembly of the lower layer are invisible; the higher layer sees the delivery as an Erlang $f$, ( $f$ exponential times on per fragment). In Figure 8(c), $B_{2}$ Erlang services must be completed to deliver a high layer packet. Since one Erlang-h service is composed of the sum of $h$ exponential random variables, delivery of a high layer packet in the model of Figure $8(c)$ takes $h B_{2}$ exponential delays; thus the model of Figure $8(\mathrm{c})$ is equivalent to the model of Figure 3 when $f=h B_{2}$. Casting the problem in this form, we can reduce the network of Figure $8(c)$ to a single queue using the approximation algorithm.

We now see why keeping the number of Erlang stages in the approximation server small is desirable. The effort to solve (11) for Figure $8(c)$ is $O\left(h W_{1} B_{2}\right)$. 


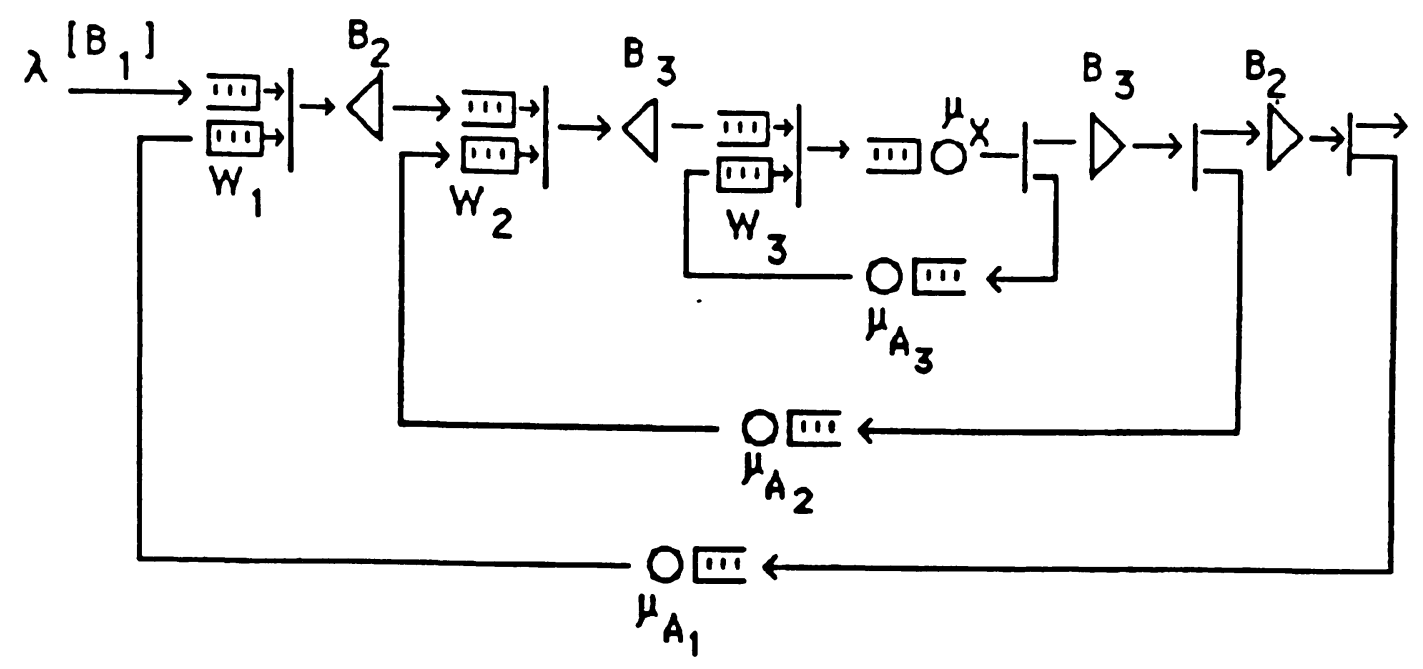

(8) - Original model

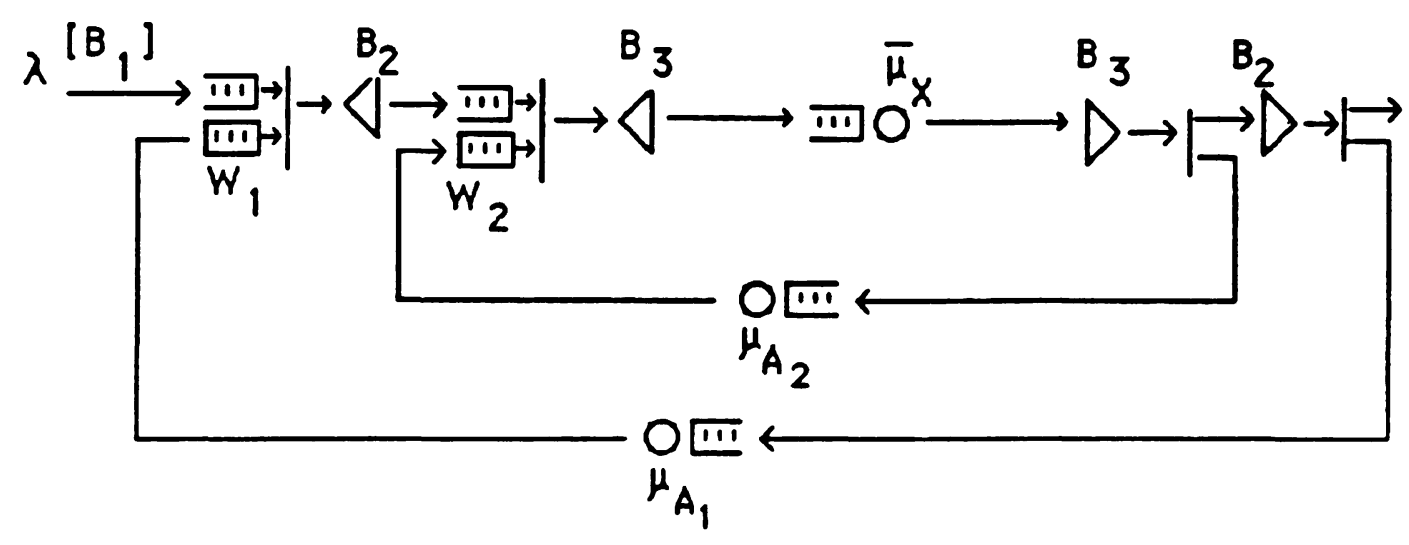

(b) - lowest level reduced

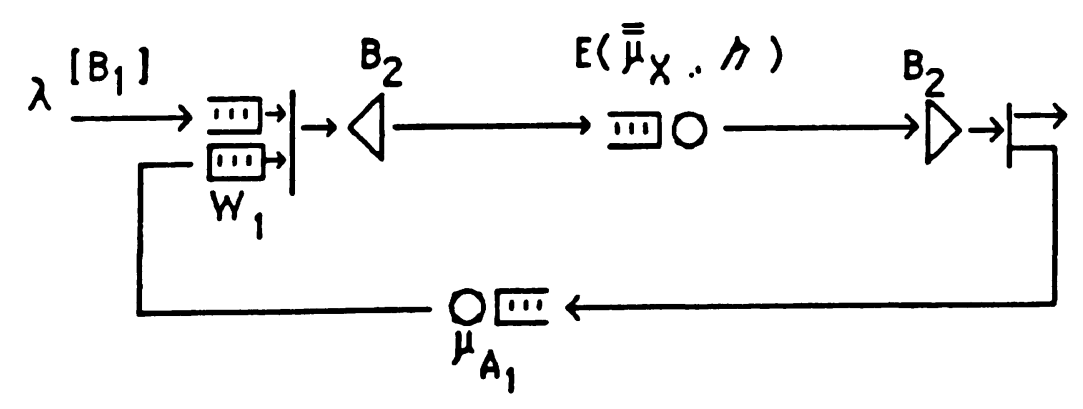

(c) - two lowest levels reduced

Fiqure 8 - Three levels of slidina window flow control 
Table 1 - Data Set Parameters for Validation of Queueing Model Shown in Figure 3

\begin{tabular}{|c|c|c|c|c|c|}
\hline $\begin{array}{l}\text { Data Set } \\
\text { Number }\end{array}$ & $\begin{array}{c}\lambda B f / \mu_{x} \text { as } \\
\% \text { of maximum }\end{array}$ & $\begin{array}{l}\text { Batch } \\
\text { size }\end{array}$ & $\begin{array}{l}\text { Window } \\
\text { size }\end{array}$ & Frag & $\left(\frac{\mu_{x}}{f}\right) \mu_{A}$ \\
\hline $\begin{array}{c}1 \\
2 \\
3 \\
4 \\
5 \\
6 \\
7 \\
8 \\
9 \\
10 \\
11 \\
12 \\
13 \\
14 \\
15 \\
16 \\
17 \\
18 \\
19 \\
20 \\
21 \\
22 \\
23 \\
24 \\
25 \\
26 \\
27 \\
28 \\
29 \\
30 \\
31 \\
32\end{array}$ & $\begin{array}{l}22 \% \\
26 \% \\
20 \% \\
20 \% \\
17 \% \\
17 \% \\
24 \% \\
35 \% \\
32 \% \\
48 \% \\
79 \% \\
67 \% \\
62 \% \\
70 \% \\
62 \% \\
60 \% \\
69 \% \\
61 \% \\
80 \% \\
67 \% \\
62 \% \\
96 \% \\
81 \% \\
93 \% \\
85 \% \\
98 \% \\
84 \% \\
80 \% \\
89 \% \\
82 \% \\
86 \% \\
85 \%\end{array}$ & $\begin{array}{l}3 \\
3 \\
3 \\
3 \\
3 \\
3 \\
3 \\
8 \\
8 \\
8 \\
3 \\
3 \\
3 \\
3 \\
3 \\
3 \\
3 \\
3 \\
6 \\
6 \\
6 \\
6 \\
6 \\
3 \\
3 \\
3 \\
3 \\
3 \\
5 \\
5 \\
5 \\
5\end{array}$ & $\begin{array}{c}2 \\
2 \\
4 \\
15 \\
2 \\
4 \\
2 \\
2 \\
4 \\
2 \\
2 \\
4 \\
8 \\
2 \\
4 \\
8 \\
2 \\
4 \\
2 \\
4 \\
8 \\
2 \\
4 \\
4 \\
8 \\
2 \\
4 \\
8 \\
4 \\
8 \\
2 \\
4\end{array}$ & $\begin{array}{r}2 \\
2 \\
2 \\
2 \\
4 \\
4 \\
16 \\
2 \\
2 \\
6 \\
2 \\
2 \\
2 \\
4 \\
4 \\
4 \\
10 \\
10 \\
2 \\
2 \\
2 \\
5 \\
5 \\
2 \\
2 \\
8 \\
8 \\
8 \\
2 \\
2 \\
5 \\
5\end{array}$ & $\begin{array}{r}.43 \\
.83 \\
.43 \\
.43 \\
.23 \\
.23 \\
.06 \\
.45 \\
.45 \\
.15 \\
.83 \\
.83 \\
.83 \\
.63 \\
.63 \\
.63 \\
.63 \\
.63 \\
.83 \\
.83 \\
.83 \\
1.25 \\
1.25 \\
.94 \\
.94 \\
.75 \\
.75 \\
.75 \\
.93 \\
.93 \\
.70 \\
.70\end{array}$ \\
\hline
\end{tabular}




\section{APPROXIMATION RESULTS}

The approximation algorithm was evaluated for 32 data sets of networks with the structure of Figure 3. The parameters for these data sets are shown in Table 1. In each case the approximate results were compared to exact solutions obtained numerically. The numerical procedure for these models required working with matrices of the order of $f$ BW, so there is a practical limit on the range of parameters which could be tested. Within these limits, the system utilization, (the second column of Table 1 shows the offered load as a percentage of the maximum possible throughput), the window size, $f$, and the ratio of high layer packet transmission time to acknowledgment transmission time, $\left(\frac{\mu_{x}}{f}\right) / \mu_{A}$ were varied.

The numerical procedure used was Neuts' matrix-geometric algorithm, [21]. Due to the highly regular structure of the rate equations (1), considerable computational efficienciescan be effected. In a straight-forward application of the matrix-geometric algorithm, a matrix of dimension IBW would need to be inverted. This matrix has a block structure, and its inverse can be evaluated directly in terms of the inverse of an $W$ sized block. The smaller block also has a regular structure, and its inverse can be written without recourse to a numerical procedure. The algorithm was written in PASCAL, and the abstract data typing features of that language facilitated the block structure representation of the rate matrix.

Figure 9 shows the relative error in mean number of high layer packets in the system vs. the predicted probability that the acknowledgment queue is full. The probability of having the acknowledgment queue full is a measure of how far from a true Erlang distribution the transmission time distribution will be. The percent errors are fairly low. In addition to the errors introduced by not having an Erlang transmission distribution, there are also errors due to approximating a system with $f>5$ by an Erlang-3. Figure 10 shows the relative error in predicted mean vs. probability that the acknowledgment queue is full, for those data sets where $f=2$. (Due to the limitations of the numerical procedure this is the only value of $f$ for which many data points were calculated). In this case, we see that the errors are roughly correlated with the probability of having the acknowledgment queue full. 


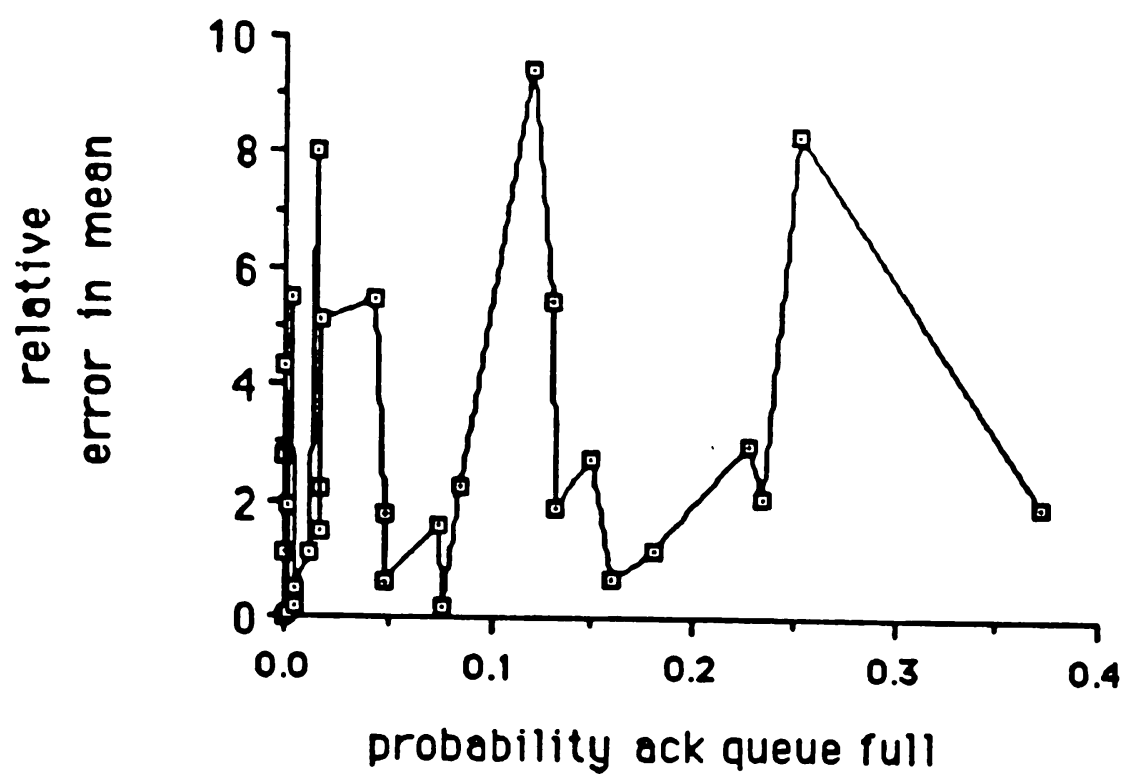

Figure 9 - Relative Error of the Mean Number of High Loyer Pockets vs.

Probobility Ack. Queue is Full

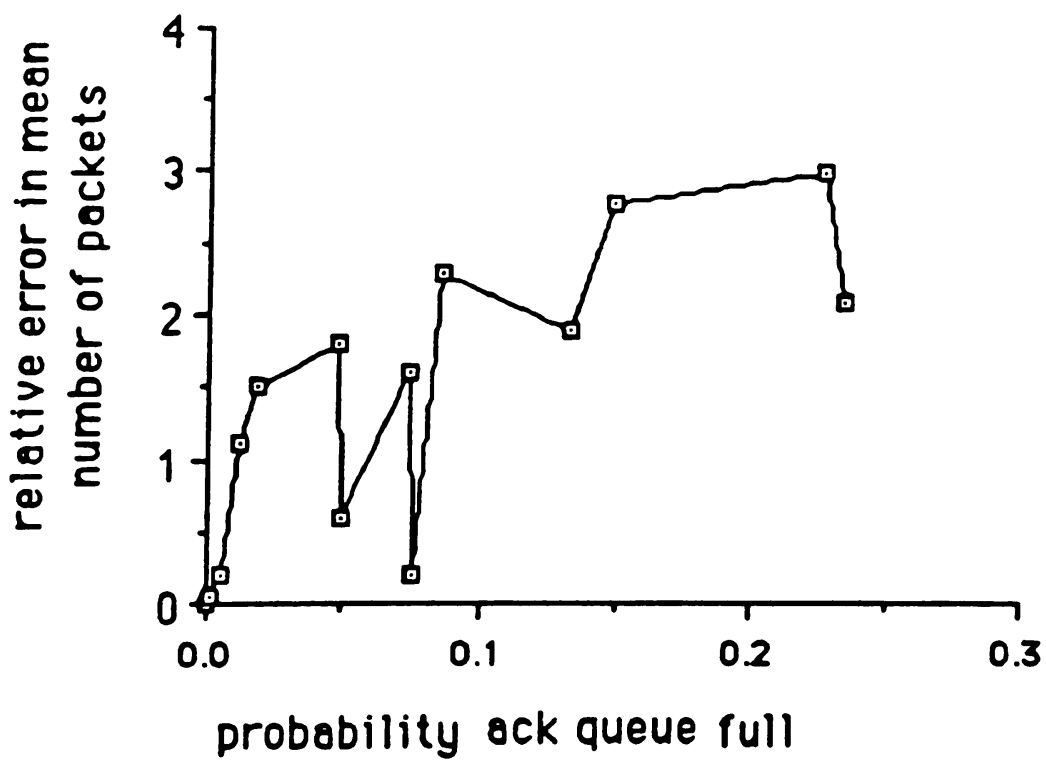

Figure 10 - Relotive Error of the Mean Number of High Layer Packets vs. Probability Ack. Queue is Full, for $\mathrm{f}=2$ 
While errors due to Erlang "fit" problems cloud the picture, it is generally true that for a given f. high utilization, high ratio $\left(\frac{\mu_{x}}{f}\right) / \mu_{A}$ and small window size will be the cases with the worst performance, having relative errors from $5 \%$ to $10 \%$. This observation matches that of previous studies, [15].

Table 2 compares the actual values for $P_{i}$ against the values from the approximating queve for the data sets of Table 1. For each data set the maximum absolute error over all $i$ and the maximum relative error for states with probability greater than 0.001 are shown. The results are fairly good. In Table 2 we also show the relative error at the value of $i$ for which the maximum absolute error was observed, and the absolute error for the value of $i$ where the maximum relative error was observed.

Figure 11 shows the predicted and actual $P$, probabilities for data set 23 , which was one of the poorest predictions, having a maximum deviation of 0.05 .

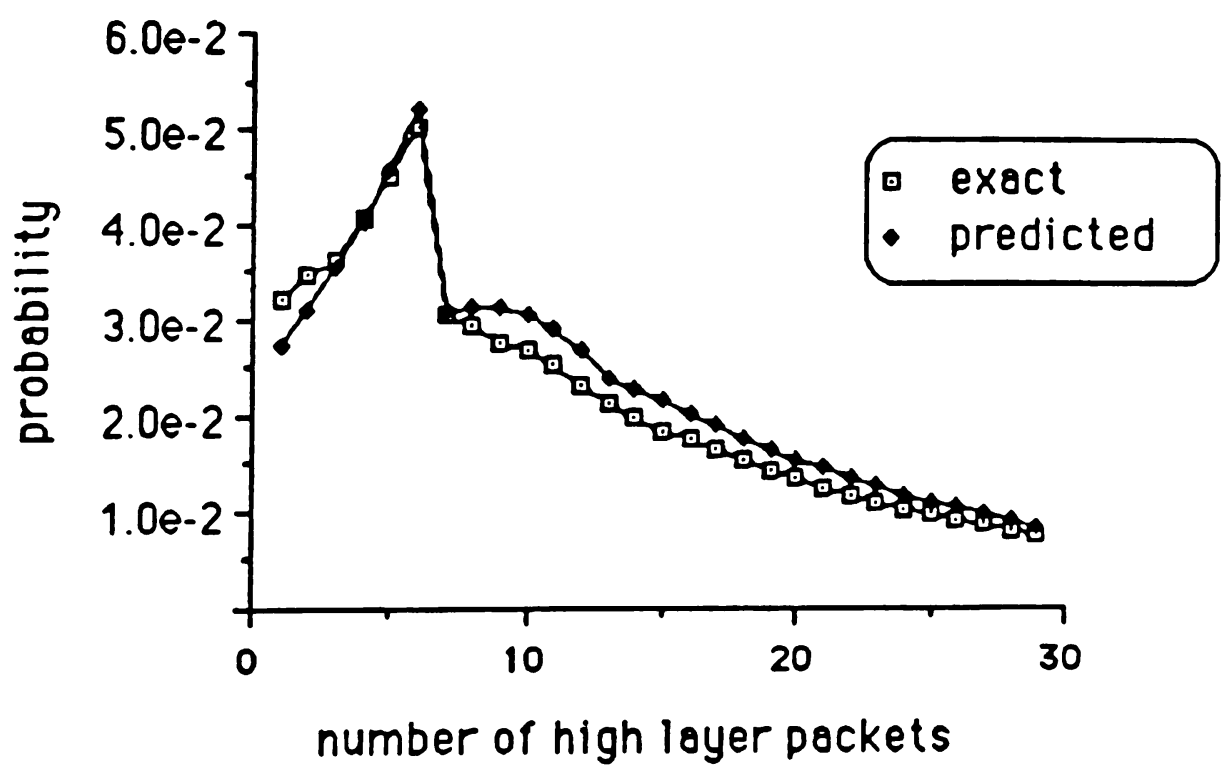

Figure 11 - Predicted vs. Actual Probability Distribution of the Number of High Layer Packets for Data Set 23 
Table 2 - Predicted vs. Actual $P_{\mathbf{i}}$

\begin{tabular}{|c|c|c|c|c|}
\hline $\begin{array}{l}\text { Data Set } \\
\text { Number }\end{array}$ & $\begin{array}{c}\text { Maximum } \\
\text { Absolute Error }\end{array}$ & $\begin{array}{l}\text { Corresp. } \\
\text { rel. error }\end{array}$ & $\begin{array}{l}\text { Maximum } \\
\text { rel.error * }\end{array}$ & $\begin{array}{l}\text { Corresp. } \\
\text { Abs. Error }\end{array}$ \\
\hline $\begin{array}{c}1 \\
2 \\
3 \\
4 \\
5 \\
6 \\
7 \\
8 \\
9 \\
10 \\
11 \\
12 \\
13 \\
14 \\
15 \\
16 \\
17 \\
18 \\
19 \\
20 \\
21 \\
22 \\
23 \\
24 \\
25 \\
26 \\
27 \\
28 \\
29 \\
30 \\
31 \\
32\end{array}$ & $\begin{array}{ll}2.98 & e-03 \\
8.45 & e-03 \\
6.2 & e-05 \\
1.8 & e-10 \\
7.0 & e-04 \\
4.3 & e-04 \\
1.5 & e-03 \\
1.8 & e-03 \\
1.6 & e-04 \\
6.5 & e-04 \\
7.0 & e-03 \\
1.4 & e-02 \\
4.1 & e-03 \\
9.5 & e-03 \\
4.0 & e-03 \\
4.4 & e-03 \\
1.3 & e-03 \\
6.6 & e-03 \\
5.0 & e-03 \\
1.4 & e-03 \\
6.1 & e-03 \\
3.5 & e-03 \\
5.0 & e-02 \\
9.2 & e-03 \\
1.3 & e-02 \\
2.1 & e-03 \\
7.9 & e-03 \\
3.0 & e-03 \\
1.3 & e-02 \\
1.4 & e-02 \\
4.8 & e-03 \\
2.1 & e-03\end{array}$ & $\begin{array}{c}5.1 \% \\
12.1 \% \\
0.008 \% \\
0.0 \% \\
1.4 \% \\
0.8 \% \\
2.0 \% \\
4.9 \% \\
0.02 \% \\
1.3 \% \\
3.1 \% \\
3.7 \% \\
1.0 \% \\
3.0 \% \\
1.0 \% \\
3.4 \% \\
3.9 \% \\
5.1 \% \\
10.0 \% \\
4.0 \% \\
1.5 \% \\
7.2 \% \\
20.3 \% \\
11.2 \% \\
7.6 \% \\
7.4 \% \\
4.6 \% \\
3.9 \% \\
10.4 \% \\
6.8 \% \\
3.2 \% \\
1.4 \%\end{array}$ & $\begin{array}{c}5.1 \% \\
12.1 \% \\
0.1 \% \\
0.0 \% \\
14.1 \% \\
13.5 \% \\
34.8 \% \\
4.9 \% \\
0.48 \% \\
10.4 \% \\
19.1 \% \\
8.1 \% \\
1.8 \% \\
17.1 \% \\
26.7 \% \\
26.4 \% \\
37.9 \% \\
46.3 \% \\
10.0 \% \\
5.25 \% \\
2.4 \% \\
9.5 \% \\
20.3 \% \\
18.3 \% \\
11.0 \% \\
9.4 \% \\
15.5 \% \\
22.7 \% \\
10.4 \% \\
6.8 \% \\
4.7 \% \\
6.3 \%\end{array}$ & $\begin{array}{l}2.98 \mathrm{e}-03 \\
8.45 \mathrm{e}-03 \\
4.7 \mathrm{e}-06 \\
1.8 \mathrm{e}-10 \\
4.0 \mathrm{e}-04 \\
3.8 \mathrm{e}-04 \\
3.6 \mathrm{e}-04 \\
1.8 \mathrm{e}-03 \\
3.2 \mathrm{e}-05 \\
1.1 \mathrm{e}-04 \\
2.2 \mathrm{e}-04 \\
9.5 \mathrm{e}-05 \\
2.0 \mathrm{e}-04 \\
1.9 \mathrm{e}-04 \\
2.8 \mathrm{e}-04 \\
3.2 \mathrm{e}-04 \\
3.9 \mathrm{e}-04 \\
5.4 \mathrm{e}-04 \\
5.0 \mathrm{e}-03 \\
1.5 \mathrm{e}-03 \\
2.3 \mathrm{e}-04 \\
1.4 \mathrm{e}-03 \\
5.0 \mathrm{e}-02 \\
1.9 \mathrm{e}-04 \\
1.2 \mathrm{e}-04 \\
9.7 \mathrm{e}-04 \\
1.6 \mathrm{e}-04 \\
2.5 \mathrm{e}-04 \\
1.3 \mathrm{e}-02 \\
1.4 \mathrm{e}-02 \\
1.8 \mathrm{e}-03 \\
6.5 \mathrm{e}-05\end{array}$ \\
\hline
\end{tabular}

- taken from states with actual probability greater than 0.001 . 
Table 3 - Simulation Data Set Parameters for Validation

of the Queueing Model shown in Figure 2

Simulation

Data Set

Number

\begin{tabular}{|c|c|c|c|c|c|c|c|c|}
\hline Number & $B_{2}$ & $B_{1}$ & $\lambda$ & $\mu_{x}$ & $\mu_{A_{1}}$ & $\mu_{A_{2}}$ & $W_{1}$ & $W_{2}$ \\
\hline $\begin{array}{l}1 a \\
1 b \\
1 c \\
1 d\end{array}$ & 1 & 1 & 10.0 & 19.8 & 20.0 & 19.0 & $\begin{array}{r}4 \\
4 \\
16 \\
16\end{array}$ & $\begin{array}{l}3 \\
8 \\
3 \\
8\end{array}$ \\
\hline $\begin{array}{l}2 a \\
2 b \\
2 c \\
2 d\end{array}$ & 2 & 1 & 5.0 & 19.8 & 20.0 & 19.0 & $\begin{array}{r}4 \\
4 \\
16 \\
16\end{array}$ & $\begin{array}{l}3 \\
8 \\
3 \\
8\end{array}$ \\
\hline $\begin{array}{l}2 \mathrm{e} \\
2 \mathrm{f} \\
2 \mathrm{~g} \\
2 \mathrm{~h} \\
\end{array}$ & 5 & 1 & 2.0 & 19.8 & 20.0 & 19.0 & $\begin{array}{r}4 \\
4 \\
16 \\
16 \\
\end{array}$ & $\begin{array}{l}3 \\
8 \\
3 \\
8 \\
\end{array}$ \\
\hline $\begin{array}{l}3 a \\
3 b \\
3 c \\
3 d\end{array}$ & 1 & 3 & 4.5 & 20.0 & 22.0 & 21.0 & $\begin{array}{l}3 \\
8 \\
3 \\
8\end{array}$ & $\begin{array}{l}3 \\
3 \\
8 \\
8 \\
\end{array}$ \\
\hline $\begin{array}{l}3 e \\
3 f \\
3 g \\
3 h \\
\end{array}$ & 1 & 8 & 2.0 & 50.0 & 55.0 & 53.0 & $\begin{array}{l}3 \\
8 \\
3 \\
8 \\
\end{array}$ & $\begin{array}{l}3 \\
3 \\
8 \\
8 \\
\end{array}$ \\
\hline $\begin{array}{l}4 a \\
4 b \\
4 c \\
4 d \\
\end{array}$ & 2 & 3 & 2.25 & 20.0 & 22.0 & 21.0 & $\begin{array}{l}3 \\
8 \\
3 \\
8 \\
\end{array}$ & $\begin{array}{l}3 \\
3 \\
8 \\
8 \\
\end{array}$ \\
\hline $\begin{array}{c}4 e \\
4 f \\
4 g \\
4 h\end{array}$ & 2 & 8 & 1.0 & 50.0 & 55.0 & 53.0 & $\begin{array}{l}3 \\
8 \\
3 \\
8 \\
\end{array}$ & $\begin{array}{l}3 \\
3 \\
8 \\
8\end{array}$ \\
\hline $\begin{array}{l}4 i \\
4 j \\
4 k \\
41\end{array}$ & 5 & 3 & 0.9 & 20.0 & 22.0 & 21.0 & $\begin{array}{l}3 \\
8 \\
3 \\
8 \\
\end{array}$ & $\begin{array}{l}3 \\
3 \\
8 \\
8 \\
\end{array}$ \\
\hline $\begin{array}{l}4 m \\
4 n \\
40 \\
4 p\end{array}$ & 5 & 8 & 0.4 & 50.0 & 55.0 & 53.0 & $\begin{array}{l}3 \\
8 \\
3 \\
8\end{array}$ & $\begin{array}{l}3 \\
3 \\
8 \\
8\end{array}$ \\
\hline
\end{tabular}




\section{Multi-layer Simulation vs. Approximation Results}

Above we compared the results of the approximation algorithm of Section III to exact results for a model with the structure of Figure 2. The comparisons of the approximate results to exact results assumes that the transmission queue is an exact representation of the lower layer network, and thus allows us to evaluate the approximation algorithm apart from errors in the lower layer approximation. We next evaluated the performance of the hierarchical method.

We performed the hierarchical reduction for 36 examples with the structure of Figure 2 . In each case the approximation algorithm was used to reduce the lower layer sliding window control shown in Figure 1 to a single queue, assuming arrivals at the rate $\lambda B_{2}$. In this case the lower layer approximation is simplified since there is no fragmentation inside the lower layer window flow control. The resulting approximation queue was then used as the transmission queue in a network with the structure of Figure 3, and this latter network was analyzed by the approximation algorithm.

The data set parameters used are shown in Table 3 . We attempted to use enough data sets to examine the various possible relationships between $W_{1}, W_{2}, B_{1}$, and $B_{2}$.

Due to the large state space associated with an exact probabilistic model of Figure 2, we are not able to generate exact solutions against which to compare the approximation results. We thus constructed a simulation model of the network in Figure 2, with the rates, window sizes, and amount of fragmentation as parameters. We used the results from a simulation model as a basis of comparison for the approximation results. For each data set in Table 3, a number of independent replications of the simulation were run. Each replication began with no packets in the system, and all tokens at both layers in the token queve. Each replication was terminated after 500 high layer packets were delivered. We calculated the mean number of high layer packets in the system for each replication, and from these values calculated an approximate $95 \%$ confidence interval for the mean number of high layer packets in the system. 
The comparison of the simulation mean number of high layer packets in the system and the predicted mean number from the hierarchical approximation is shown in Table 4 We see that with the exception of cases $3 a, 3 c, 3 f, 3 h, 4 a$, and $4 c$, the approximate means lie within the $95 \%$ confidence intervals.

We also used the simulation data to construct point estimates of the probability distribution of the number of high layer packets in the system, along with the associated approximate $95 \%$ confidence intervals for these estimates. Figure 12 shows a comparison of the probability distribution of high layer packets in the system obtained using simulation against the probability distribution generated by the approximation algorithm. Figure 12 is for case $4 \mathrm{~m}$, and was selected as being typical.

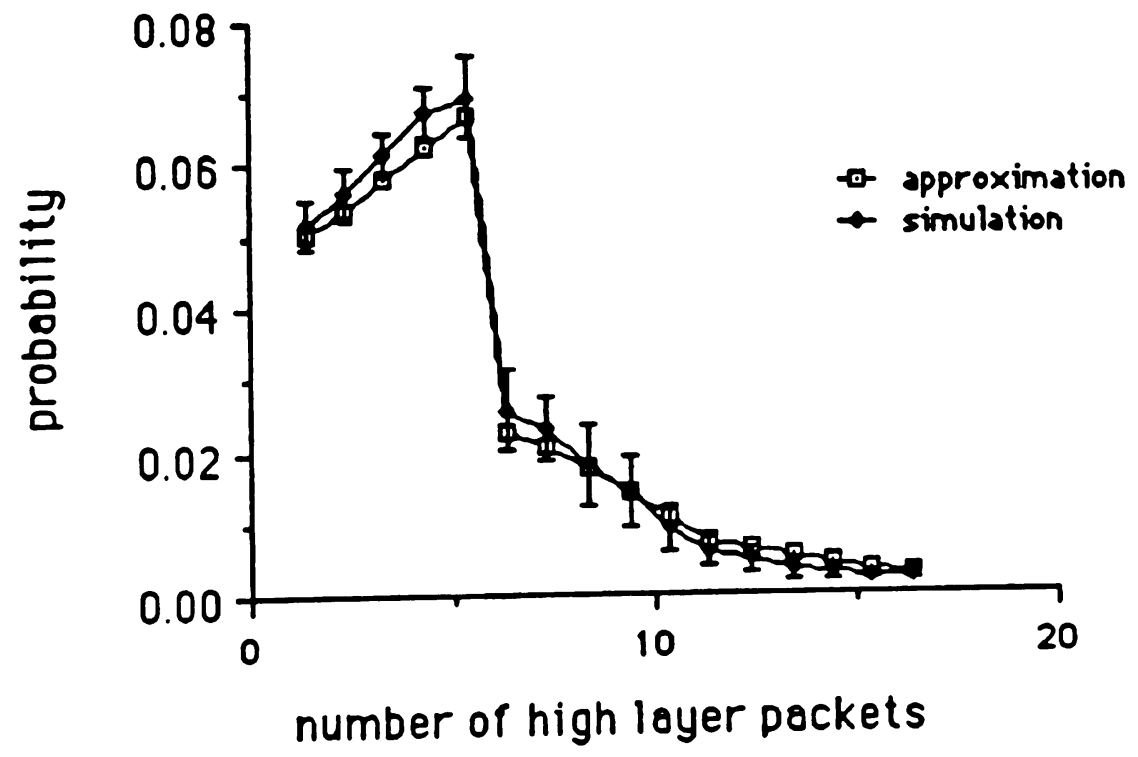

Figure 12 - Comparison of Simulation and Approximation Probability Distributions of the Number of High Layer Packets for Data Set $4 \mathrm{~m}$

We also experimented with a state-dependent flow-equivalent server approach, developed by Fdida, Perros, and Wilk. [19]. The authors developed this procedure assuming single Poisson arrivals at rate $\lambda$ : we generalized the procedure to batch arrivals. (as in 114]). and tested the procedure against sample data sets for the model of Figure 3 with $f=1$. These results were then compared to the exacl solution computed by a matrix-geometric procedure. 
Table 4-Comparison of Approximate and Simulation Means for the Data Sets Given in Table 3

\begin{tabular}{c|c|c|c|}
$\begin{array}{c}\text { Data } \\
\text { Set }\end{array}$ & \multicolumn{1}{c}{$\begin{array}{c}\text { Approx. } \\
\text { mean }\end{array}$} & \multicolumn{1}{c}{$\begin{array}{c}\text { Simulation } \\
\text { mean }\end{array}$} & $\begin{array}{c}95 \% \text { conf. } \\
\text { interval }\end{array}$ \\
\hline & & & \\
1a & 1.64086 & 1.609 & \pm 0.3516 \\
1b & 1.182 & 1.1778 & \pm 0.1997 \\
1c & 1.4297 & 1.445 & \pm 0.2807 \\
1d & 1.0355 & 1.001 & \pm 0.1308 \\
\hline $2 \mathrm{a}$ & 2.7365 & 2.3068 & \pm 0.5234 \\
$2 \mathrm{~b}$ & 1.8230 & 1.7338 & \pm 0.3324 \\
$2 \mathrm{c}$ & 2.4025 & 2.033 & \pm 0.4543 \\
$2 \mathrm{~d}$ & 1.6075 & 1.4247 & \pm 0.2428 \\
\hline $2 \mathrm{e}$ & 6.2412 & 6.108 & \pm 1.610 \\
$2 \mathrm{f}$ & 3.9034 & 4.1997 & \pm 0.8466 \\
$2 \mathrm{~g}$ & 5.5368 & 5.4561 & \pm 1.4297 \\
$2 \mathrm{~h}$ & 3.5031 & 2.8488 & \pm 0.6891 \\
\hline $3 \mathrm{a}$ & 4.0051 & 3.2045 & \pm 0.4435 \\
$3 \mathrm{~b}$ & 1.9267 & 1.7681 & \pm 0.2595 \\
$3 \mathrm{c}$ & 3.9699 & 3.2013 & \pm 0.4440 \\
$3 \mathrm{~d}$ & 1.9115 & 1.7655 & \pm 0.2593 \\
\hline $3 \mathrm{e}$ & 0.5351 & 0.5244 & \pm 0.0286 \\
$3 \mathrm{f}$ & 0.4347 & 0.4127 & \pm 0.0194 \\
$3 \mathrm{~g}$ & 0.5351 & 0.5245 & \pm 0.0286 \\
$3 \mathrm{~h}$ & 0.4347 & 0.4127 & \pm 0.0194 \\
\hline $4 \mathrm{a}$ & 6.5422 & 5.6219 & \pm 0.8189 \\
\hline $\mathrm{b}$ & 3.1004 & 2.7855 & \pm 0.4104 \\
$4 \mathrm{c}$ & 6.4776 & 5.6043 & \pm 0.8200 \\
$4 \mathrm{~d}$ & 3.0694 & 2.7749 & \pm 0.4123 \\
\hline $4 \mathrm{e}$ & 0.8414 & 0.8300 & \pm 0.0724 \\
$4 \mathrm{f}$ & 0.6787 & 0.6452 & \pm 0.0505 \\
$4 \mathrm{~g}$ & 0.8414 & 0.8300 & \pm 0.0724 \\
$4 \mathrm{~h}$ & 0.6787 & 0.6452 & \pm 0.0505 \\
\hline $4 \mathrm{i}$ & 11.416 & 13.693 & \pm 3.1462 \\
$4 \mathrm{j}$ & 6.6247 & 7.4186 & \pm 1.4554 \\
$4 \mathrm{k}$ & 14.001 & 13.675 & \pm 3.1474 \\
$4 \mathrm{l}$ & 6.5432 & 7.3887 & \pm 1.4545 \\
\hline $4 \mathrm{~m}$ & 1.7604 & 1.6871 & \pm 0.2271 \\
$4 \mathrm{n}$ & 1.4108 & 1.3066 & \pm 0.1541 \\
40 & 1.7604 & 1.6871 & \pm 0.2271 \\
$4 \mathrm{p}$ & 1.4108 & 1.3066 & \pm 0.1541 \\
& & &
\end{tabular}


The method performed well with single arrivals, though errors were slightly higher for both predicted mean and predicted distribution compared to the algorithm in this paper. For batched arrivals, $(B>1)$, the flow-equivalence method was markedly inferior. The predicted state probabilities were monotonically decreasing, and did not reflect the true shape of the distribution. The relative errors for the mean number in the system exceeded $25 \%$ in some cases.

An explanation for the poor performance of the flow-equivalence methodology with batched arrivals is the assumption inherent in the technique that the service rate with $n$ packets in the system is the service rate of a closed system in steady-state with $\min (n, W)$ packets present. With batched arrivals this instantaneous steady-state assumption is not warranted.

\section{CONCLUSIONS}

In this paper we have presented a new procedure for approximating the behavior of a single hop network with multiple layers of sliding window flow control. The method differs from most previous research presented in the published literature in that it explicitly takes into account the fragmentation of packets which occurs in actual systems. The procedure was compared to exact numerical solutions, and found to perform well.

We see particularly that for systems with batched arrivals that the state asymptotic approach yields better results than procedures based on a flow-equivalent server approach. The flowequivalent server technique assumes an "instantaneous steady state" behavior of the network, that is, the network with a given population behaves like a closed network in steady state with that population. This technique is successful in the models where the state does not change dramatically in any one instantaneous transition. This is clearly not the case in systems with batched arrivals.

The procedure is easy to implement, and easy to solve on a computer. Its speed makes it feasible to think of including the procedure in an interactive network designer expert system, as a module to evaluate a proposed network which has a sliding window protocol. 
The procedure reports its results as the service rate and number of exponential service stages of a queue. This should make it a useful add-on to general purpose queueing network solution software packages. These packages typically cannot handle the special features of a sliding window protocol. By reducing the portions of the queueing network which have sliding window control to a single queue, the procedure approximates the sliding window sub-net by a structure which can be handled by the software package. 


\section{REFERENCES}

[1] A.S. Tanenbaum, Computer Networks, Englewood Cliffs, NJ: Prentice-Hall, Second Edition, 1988.

[2] M. Gerla and L. Kleinrock, "Flow Control: A Comparative Survey," IEEE Trans. Commun., Vol. COM-28, April 1980

[3] M.C. Pennotti and M. Schwartz, "Congestion Control in Store and Forward Tandem Links," IEEE Trans. Commun., Vol. COM-23, December 1975.

[4] A. Chaterjee, N.D. Georganas, and P.K. Verma, "Analysis of a Packet Switched Network with End to End Congestion Control and Random Routing," IEEE Trans. Commun., Vol. COM-25, December 1977.

[5] J. Labetoulle, G. Pujolle, and N. Mikou, "A Study of Flows in an X25 Environment," in Flow Control in Computer Networks, J.L. Grange and M. Gien, Eds, NY: North Holland, 1979.

[6] M. Reiser, "A Queueing Network Analysis of Computer Communication Networks with Window Flow Control," IEEE Trans. Commun. Vol. COM-27, August 1979.

[7] S. Lam, "Queueing Networks with Population Size Constraint," IBM Journal of Res. and Develop., Vol. 21, No. 4, 1977.

[8] A. Thomasian and P. Bay, "Analysis of Queueing Network Models with Population Size Constraints and Delayed Blocked Customers," in Proc. 1984 ACM SIGMETRICS Conference on Measurement and Modelling of Computer Systems, a special issue of Performance Evaluation Rev., Vol.12, No. 3, 1984.

[9] K. Goto, Y. Takahashi, and J. Hasegawa, "An Approximate Analysis of Controlled Tandem Queues," in Proc. of the Int'I Seminar on Modelling and Performance Evaluation Methodology, Paris, 1983.

[10] I.F. Akyildiz, "Performance Analysis of Computer and Communications Networks with Local and Global Window Flow Control," INFOCOM '88, pp. 400-410.

[11] L. Kleinrock and P. Kermani, "Static Flow Control in Store and Forward Computer Networks," IEEE Trans. Commun., Vol. COM-28, February 1980.

[12] M. Reiser, "Admission Delays on Virtual Routes with Window Flow Control," in Perf. of Data Commun. Systems and Their Applications, G. Pujolle, ed., NY: North Holland, 1981.

[13] A. Thomasian and P. Bay, "Performance Analysis of Window Flow Control for Multiple Virtual Routes," Proc. IEEE INFOCOM '84, San Francisco, April 9, 1984.

[14] O. Gihr and P.J. Kuehn, "Comparison of Communication Services with Connection-oriented and Connectionless Data Transmission," in Proc. Int'I. Seminar on Computer Networking and Performance Evaluation, Tokyo, September 18-20, 1985.

[15] G. Varghese, W. Chou, and A.A. Nilsson, "Queueing Delays on Virtual Circuits using a Sliding Window Flow Control Scheme," Proc. ACM SIGMETRICS Conference, Minneapolis, 1983.

[16] M. Schwartz, "Performance Analysis of the SNA Virtual Route Pacing Control," IEEE Trans. Commun., Vol. COM-30, Jan. 1982.

[17] L.C. Mitchell and D.A. Lide, "End-to-End Performance Modeling of Local Area Networks," IEEE Journal on Selected Areas in Commun., Vol. SAC-4, Sept. 1986. 
[18] M. Murata and H. Takagi, "Two-Layer Modeling for Local Area Networks," IEEE Trans. Commun., Vol. COM-36, Sept. 1988.

[19] S. Fdida, H. Perros, and A. Wilk, "Semaphore Queues: Modelling Multi-Layered Window Flow Control Mechanisms," IEEE Trans. Commun., (to appear).

[20] R. Marie, "Calculating Equilibrium Probabilities for $\lambda(n) / C_{k} / 1 / N$ Queues," in Proceedings of Performance '80, Toronto, 1980.

[21] M. Neuts, Matrix-Geometric Solutions in Stochastic Models, Johns Hopkins University Press. Baltimore, Md., 1981. 